Abstract: Tissue engineering is one of the most exciting and rapidly growing areas in biomedical engineering that offers vast potential for changing traditional approaches to meeting many pharmaceutics and critical health care needs. Currently the bottle-neck area in this multidisciplinary field appears to be materials and fabrication technology for the design of artificial extracellular matrices/scaffolds that support culturing and growth of new tissue. We have shown that stable relief structures can be created and maintained in the bulk of ice by continuous s canning with computer-guided IR $\mathrm{CO}_{2}$ laser. The optimal laser beam intensity and fluence rate distribution within the ice sample, as well as the rate of scanning were estimated based on the Monte Carlo model utilized physical/optical properties of ice. The results of numerical simulation are agreed well with the observed experimental results of thermo-coupling measurements and obtained microscopic images.

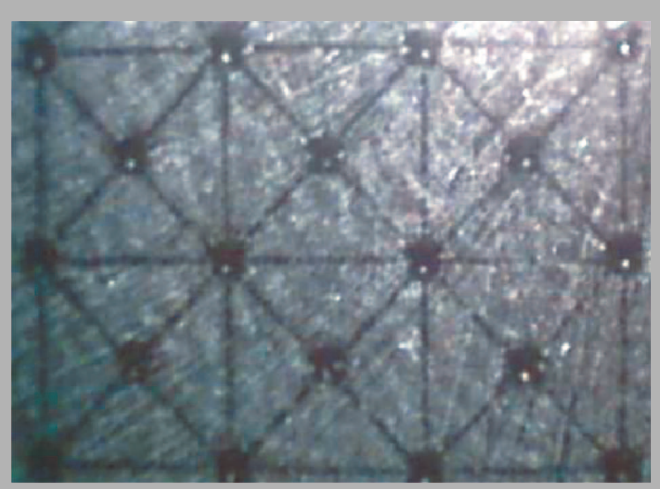

The dimples connected by channels welded at the ice surface by the repetitive scanning with IR $\mathrm{CO}_{2}$ laser

\title{
Laser ice scaffolds modeling for tissue engineering
}

\author{
I.V. Meglinski, ${ }^{1, *}$ M. Varejka, ${ }^{2}$ A.C. Woodman, ${ }^{2}$ A.P.F. Turner, ${ }^{2}$ and S.A. Piletsky ${ }^{2}$ \\ ${ }^{1}$ School of Engineering, Cranfield University, Cranfield, MK43 0AL, UK \\ ${ }^{2}$ Institute of Bio-Science and Technology, Cranfield University, Silsoe, MK45 4DT, UK
}

Key words: tissue engineering; ice scaffolds modeling; laser ice welding

PACS: $42.62 . \mathrm{Be}, 87.80 . \mathrm{Rb}, 87.80 .-\mathrm{y}$

Tissue engineering is one of the most exciting and rapidly growing areas in biomedical engineering that offers vast potential for changing traditional approaches to meeting many pharmaceutics and critical health care needs [1]. Currently the bottle-neck area in this multidisciplinary field appears to be materials and fabrication technology for the design of artificial extra-cellular matrices/scaffolds that support culturing and growth of new tissue. Most of approaches in tissue engineering, including design of the scaffolds, have empirical, "try-and-test" nature, and identification of the parameters of "ideal" scaffold is highly required [2]. This explains considerable grow of interest to the computational and physical modeling of scaffolds parameters. These parameters are shape and architecture, porosity and branching, transport properties, structural integrity and degradation process.

The rapid prototyping of physical models of scaffolds can be performed by using solid free-form fabrica- tion (SFF), which is a variation of an advanced computeraided-design (CAD) approach $[3,4]$. This technology often applies laser based sintering or stereo-lithography when 3-D polymer structures are formed as a result of partial decomposition of the polymer [5] or continuous layered deposition of polymer structures [6]. The problems associated with this approach include high cost, limited selection of polymerisable biodegradable materials, relatively poor resolution and still too long time required to design and test any particular model which prevents fast optimization of scaffold engineering process [7]. Here we present a new simple approach for rapid express modeling and designing scaffolds in the ice formed with computerguided $\mathrm{CO}_{2}$ IR laser. The laser is used to create complex pattern combined series of channels, cavities, circles, etc. at the ice surface. As a next step which follows modeling, this patterning can be used to create permanent "negative" micro-architecture by filling the ice cavities, dents

\footnotetext{
*Corresponding author: e-mail: i.meglinski@cranfield.ac.uk
} 


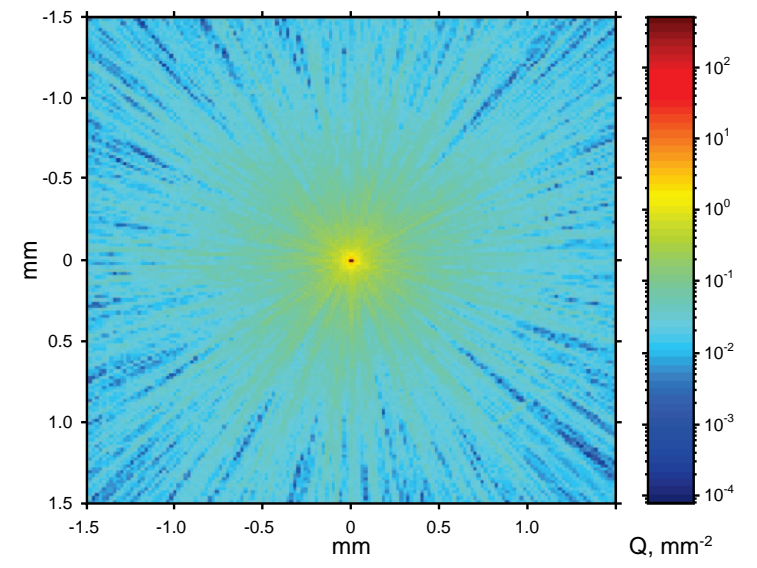

Figure 1 (online color at www.lphys.org) The ice surface energy distribution calculated by the MC technique

and pores with a polymeric solution. The polymeric solution solidifies later whereas the ice melts that creates required porous scaffold. The pattern welded with laser can be changed easily and rapidly that permits fast optimization of flow and transport of polymeric solution and/or any other liquids of interest. The optimal laser beam intensity and the rate of scanning were estimated based on the ice physical/optical properties [8-11] and modeling experiment describing below.

Experimental set-up. The profiling of ice surface is performed using the electrical galvanometer scanning based on the Fenix laser system (SYNRAD). The laser beam generated by a high power $30 \mathrm{~W} \mathrm{CO}_{2}$ laser has been delivered to $X$ scanning mirror mounted about $45^{\circ}$ position. This deflects the beam at right angles to initial line of radiation travel and upwards onto the $Y$ mirror. The $Y$ mirror automatically fed a sine wave takes the line automatically drawn by the $X$ mirror and moves it at the sample surface. When the $Y$ scanner fed with the same sine wave as the $X$ scanner, the projected image would be a line at $45^{\circ}$ angle. Using the $X-Y$ electrical galvanometer scanning system fed from analogue oscillator circuits, the position of the beam can be controlled so as to allow for the projection of laser patterns at the sample surface. This scanning system uses to scan focused laser beam (focal length $80 \mathrm{~mm}$ ) through the ice sample producing complex geometrical shapes of $25 \mathrm{~mm}$ length and $250-300 \mathrm{~m}$ width with the resolution determined by the laser spot size $(50 \mathrm{~m})$ and irradiation intensity. Observation of the artwork created in ice is done with a Digital Blue TM Camera. The temperature changes in the ice were measured by thermocoupling (TME electronics, UK) frozen at the ice surface. The transport of liquid within the channels was achieved by creating two apertures, one of which was filled with dye solution (Melbda's blue, $0.10 \mathrm{~g} / \mathrm{ml}$ ). The scanning laser beam intersects these apertures creating gradient of the dye in the channels observed with a Digital Blue TM Camera.

A simplified physical/optical model of ice laser beam welding has been used. For the sake of simplification we assume that the ice sample plate is homogeneous and the flux of ice melted is linearly proportional to the part of the absorbed laser intensity left after subtraction of the heat conduction losses. Optical properties of ice, i.e. absorption coefficient $\left(\mu_{a}=0.04 \mathrm{~mm}^{-1}\right)$, scattering coefficient $\left(\mu_{s}=0.2 \mathrm{~mm}^{-1}\right)$, anisotropy factor $(g=0.9)$ and refractive index $(n=1.33)$ were chosen follow the results of the ice properties studies reported earlier [8-11]. The estimation of propagation of laser radiation and its fluence rate distribution within and under the surface of the sample has estimated based on the Monte Carlo (MC) technique developed in-house. This MC approach combines the statistical weight scheme and effective optical paths simulation. The details of the technique are justified everywhere [12, 13] and are not presented here. The results of simulation presented in Fig. 1 show the fluence rate distribution at the surface of ice sample. The figure clearly illustrates, that focused laser beam produces maximum localization of energy $Q$ [a.u. $/ \mathrm{mm}^{2}$ ] with the in the area $250-300 \mathrm{~mm}^{2}$.

Knowing the specific density $\rho$ and heat capacity of ice $C\left(\rho_{i c e}=920 \mathrm{~kg} \cdot \mathrm{m}^{-3}\right.$ and $C_{\text {ice }}=2.17 \times$ $\left.10^{3} \mathrm{~J} \cdot \mathrm{kg}^{-1} \cdot \mathrm{K}^{-1}\right)$ and water $\left(\rho_{\text {water }}=999.87 \mathrm{~kg} \cdot \mathrm{m}^{-3}\right.$ and $C_{\text {water }}=4.2 \times 10^{3} \mathrm{~J} \cdot \mathrm{kg}^{-1} \cdot \mathrm{K}^{-1}$ ) the optimal power of laser radiation and rate of scanning for a single vessel were estimated as $1-2 \mathrm{~W}$ and $100 \mathrm{~mm} \cdot \mathrm{s}^{-1}$, respectively. These parameters are well agreed with the results of the experiment. Fig. 2 show the example of channels and depressions drawn on the ice surface using a focused laser beam as discussed above. Five channels $2 \mathrm{~mm}$ long and $200 \mathrm{~m}$ width have been drawn after five passes. The picture was taken with a Digital Blue camera with a 10 times engrossment lens.

Practically any type of geometric features - dimples and/or channels, as well as any other relief with dimensions $0.2-100 \mathrm{~mm}$ could be accurately created in the ice within a minute (Fig. 2). Created features could be maintained in the ice during prolonged period of time by continuing scanning at fraction of original intensity (typically 50). What is important that liquid flow can be achieved (and controlled by gradient of concentration, pressure or current) through the system of channels created in ice as shown in Figs. 2a and 2b. This is important for proper functioning of tissue scaffolds.

We have made an attempt to analyze the temperature created in local area by scanning of laser beam with different intensity. To achieve this, thermo-coupling has been frozen in the sample of the ice and ten adjusted channels were drawn through the exposed area of the wires. As can be seen from the Fig. 3a the local temperature can be increased by $1-6{ }^{\circ} \mathrm{C}$ by repetitive scanning of low power laser beam. It should be pointed out that this temperature is an average temperature that corresponds to the quite large size of thermo-coupling measuring area. The real temperature in the channel or in the dimple created in ice is several degrees higher.

The typical profile of the temperature change followed one scan is shown in Fig. 3b. Apparently fast rise in the temperature after flash melts the ice, which exists in liquid 


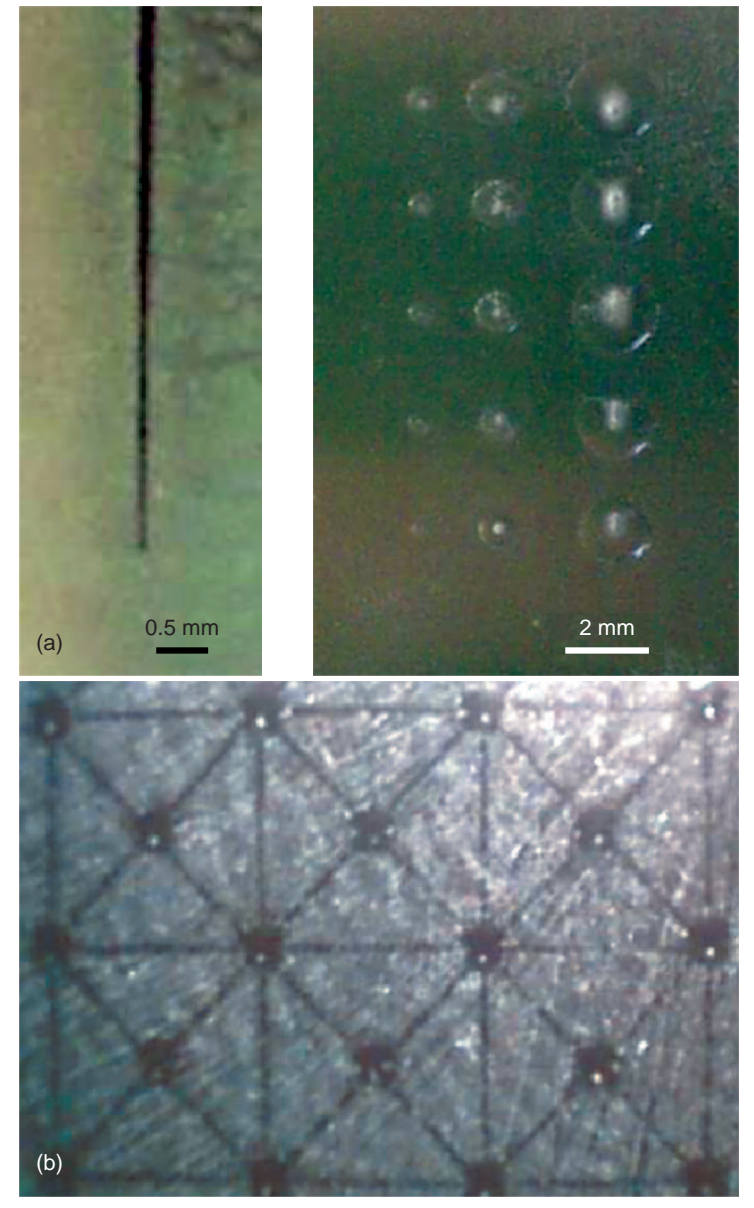

Figure 2 (online color at www.lphys.org) Examples of patterns produced by the IR $\mathrm{CO}_{2}$ laser with the electrical galvanometer scanning system described below: (a) - channel and dimples, (b) - dimples connected by channels. The patterns formed at the ice surface by repetitive scanning (100 times) of $0.6 \mathrm{~W} \mathrm{CO}_{2}$ laser at $100 \mathrm{~mm} \cdot \mathrm{s}^{-1}$; depressions in the ice formed by repetitive scanning (25 times) of $1.2 \mathrm{~W} \mathrm{CO}_{2}$ laser at $100 \mathrm{~mm} \cdot \mathrm{s}^{-1}$

form for approximately $6-10$ seconds, after which the water freezes again. To maintain channels open requires continuing scanning repeated every 10 seconds.

In conclusion, we have shown that stable relief structures can be created and maintained in the bulk of ice by continuous scanning with computer-guided IR laser. The optimal laser beam intensity and fluence rate distribution within the ice sample, as well as the rate of scanning were estimated based on the MC model utilized ice physical/optical properties. The results of the numerical simulation are well agreed with the observed experimental results of thermo-coupling measurements and obtained microscopic images. Using the laser to create the mask-less artwork is an ideal process for one-of-a-kind custom piece as well as large production runs. The continuous transport of liquid through the created open channels was observed as well. The future work will be focused on the optimiza-
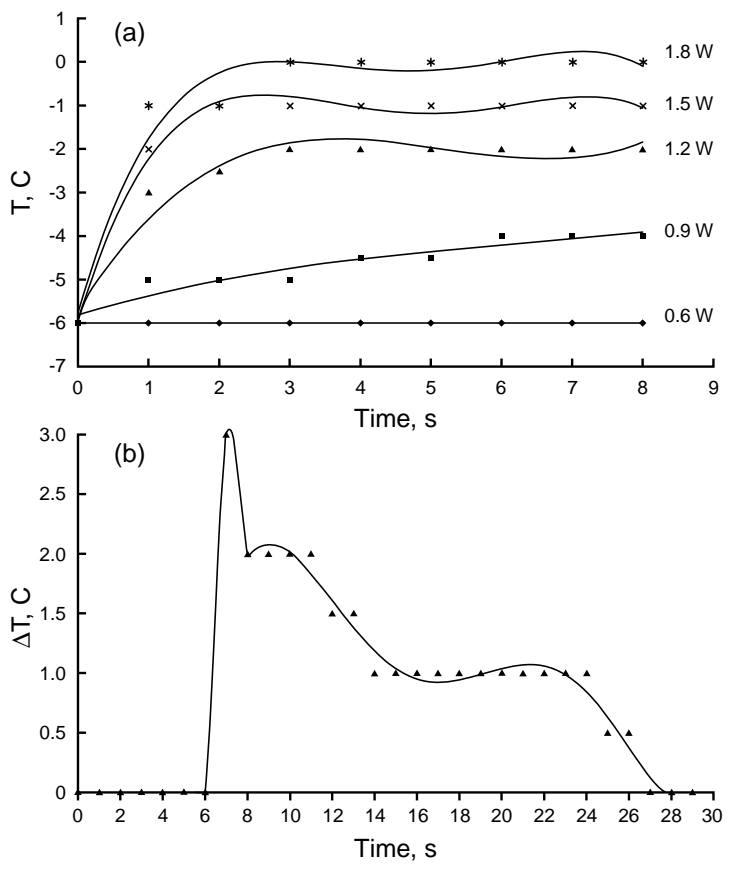

Figure 3 The temperature changes measured by frozen thermocoupling upon continuing scanning of ten adjusted channels (a) and one channel (b) drawn through the surface of the wire. The scanning speed is $100 \mathrm{~mm} \cdot \mathrm{s}^{-1}$

tion of channels transport properties and other features created in the ice.

\section{References}

[1] Trends in Tissue Engineering (Washington, DC, USA, November 20, 2002).

[2] D.W. Hutmacher, M. Sittinger, and M.V. Risbud, Trends in Biotechnol. 22, 354 (2004).

[3] D.T. Pham, R.S. Gault, Int. J. Mach. Tools Manuf. 38, 1257 (1998).

[4] S. Yang, K.-F. Leong, Z. Du, and C.-K. Chua, Tissue Eng. 8, 1 (2002).

[5] J.T. Rimell and P.M. Marquis, J. Biomed. Mater. Res. 53, 414 (2000).

[6] M.N. Cooke, J.P. Fisher, D. Dean, et al., J. Biomed. Mater. Res. 64B, 65 (2003).

[7] P.X. Ma, Materials Today 7, 30 (2004).

[8] R.G. Onstott, P. Gogineni, A.J. Gow, et al., IEEE Transact. Geosci. Remote Sensing 36, 1764 (1998).

[9] K.C. Jezek, D.K. Perovich, K.M. Golden, et al., IEEE Transact. Geosci. Remote Sensing 36, 1633 (1998).

[10] V.V. Semak, W.D. Bragg, B. Damkroger, and S. Kempka, J. Phys. D 32, L61 (1999).

[11] A. Matsunawa and V. Semak, J. Phys. D: Appl. Phys. 30, 798 (1997).

[12] D.Y. Churmakov, I.V. Meglinski, and D.A. Greenhalgh, Phys. Med. Biol. 47, 4271 (2002).

[13] I.V. Meglinski, D.Y. Churmakov, A.N. Bashkatov, et al., Laser Phys. 13, 65 (2003). 